\title{
The theory of nonequilibrium Anderson impurity model for strongly correlated electron systems
}

\author{
V.A. Moskalenko ${ }^{1,2}$, L.A. Dohotaru ${ }^{3,1}$, and D.F. Digor ${ }^{1}$ \\ ${ }^{1}$ Institute of Applied Physics, Moldova Academy of Sciences, Chisinau 2028, Moldova \\ E-mail: moskalen@theor.jinr.ru \\ ${ }^{2}$ Bogolyubov Laboratory of Theoretical Physics, Joint Institute for Nuclear Research, Dubna 141980, Russia \\ ${ }^{3}$ Technical University, Chisinau 2004, Moldova
}

Received October 15, 2014, published online March 23, 2015

\begin{abstract}
The nonequilibrium theory of strongly correlated systems is proposed theory which is grounded on the generalized Wick theorem. This theorem is employed for calculation of the thermal averages of the contour arranged products of electron operators by generalizing Keldysh formalism. Perturbation expansion is realized for Anderson impurity model in which we consider the Coulomb interaction of the impurity electrons as a main parameter of the model and the mixing interaction between impurity and conduction electrons as a perturbation. The first two approximations are used and is obtained the value of the current between one of the leads and central region of interacting electrons. The contribution of the strong correlations and of irreducible diagrams is analyzed.
\end{abstract}

PACS: 71.27.+a Strongly correlated electron systems; heavy fermions;

71.10.Fd Lattice fermion models.

Keywords: Keldysh diagram technique, nonequilibrium state, Green’s function, Anderson impurity model.

\section{Introduction}

We start with single Anderson impurity model connected to two leads named left (L) and right (R) with Hamiltonian

$$
\begin{gathered}
H=H^{0}+H_{i}, \\
H^{0}=\sum_{\lambda=L, R} \sum_{\mathbf{k} \sigma} \epsilon_{\mathbf{k} \lambda} C_{\mathbf{k} \lambda \sigma}^{+} C_{\mathbf{k} \lambda \sigma}+\sum_{\sigma} \epsilon_{d} n_{d \sigma}+U n_{d \uparrow} n_{d \downarrow}, \\
H_{i}=\sum_{\lambda=L, R} \sum_{\mathbf{k} \sigma}\left(V_{\mathbf{k} \lambda} d_{\sigma}^{+} C_{\mathbf{k} \lambda \sigma}+V_{\mathbf{k} \lambda}^{*} C_{\mathbf{k} \lambda \sigma}^{+} d_{\sigma}\right),
\end{gathered}
$$

where $d_{\sigma}$ and $C_{\mathbf{k} \lambda \sigma}$ are the annihilation operators of the dot and leads electrons, correspondingly, with spin $\sigma, \epsilon_{\mathbf{k} \lambda}$ is the leads electron energy eigenvalues, $\epsilon_{d}$ is the dot's electron on site energy, $U$ is the Coulomb repulsion, $V_{\mathbf{k} \lambda}$ is the mixing matrix elements which describe the couplings between dot and leads, $n_{\sigma}=d_{\sigma}^{+} d_{\sigma}$.

We shall use the operators $b_{\lambda \sigma}$ of the localized mode of leads conduction electrons

$$
b_{\lambda \sigma}=\sum_{\mathbf{k}} V_{\mathbf{k} \lambda} C_{\mathbf{k} \lambda \sigma}
$$

and investigate the influence of the localized electrons of impurity on this collective mode of conduction electrons.

The chemical potentials of both leads are supposed to be different and system is in nonequilibrium state. Therefore we employ the Keldysh [1-3] formalism based on the contour of the time evolution of the Fig. 1 and four local Green's functions for every of both subsystems of electrons:

$$
\begin{aligned}
& G_{\sigma \sigma^{\prime}}^{--}\left(t, t^{\prime}\right)=-i\left\langle T d_{\sigma}(t) d_{\sigma^{\prime}}^{+}\left(t^{\prime}\right)\right\rangle, \\
& G_{\sigma \sigma^{\prime}}^{-+}\left(t, t^{\prime}\right)=i\left\langle d_{\sigma^{\prime}}^{+}\left(t^{\prime}\right) d_{\sigma}(t)\right\rangle, \\
& G_{\sigma \sigma^{\prime}}^{+-}\left(t, t^{\prime}\right)=-i\left\langle d_{\sigma}(t) d_{\sigma^{\prime}}^{+}\left(t^{\prime}\right)\right\rangle, \\
& G_{\sigma \sigma^{\prime}}^{++}\left(t, t^{\prime}\right)=-i\left\langle\tilde{T} d_{\sigma}(t) d_{\sigma^{\prime}}^{+}\left(t^{\prime}\right)\right\rangle,
\end{aligned}
$$

and

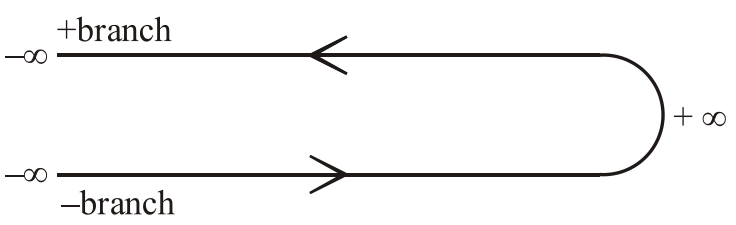

Fig. 1. The Keldysh contour of the time evolution. 


$$
\begin{aligned}
& \mathcal{G}_{\lambda \lambda^{\prime}}^{--}\left(t, t^{\prime}\right)=-i\left\langle T b_{\lambda}(t) b_{\lambda^{\prime}}^{+}\left(t^{\prime}\right)\right\rangle, \\
& \mathcal{G}_{\lambda \lambda^{\prime}}^{-+}\left(t, t^{\prime}\right)=i\left\langle b_{\lambda}^{+}\left(t^{\prime}\right) b_{\lambda}(t)\right\rangle, \\
& \mathcal{G}_{\lambda \lambda^{\prime}}^{+-}\left(t, t^{\prime}\right)=-i\left\langle b_{\lambda}(t) b_{\lambda^{\prime}}^{+}\left(t^{\prime}\right)\right\rangle, \\
& \mathcal{G}_{\lambda \lambda^{\prime}}^{++}(t, t)=-i\left\langle\tilde{T} b_{\lambda}(t) b_{\lambda^{\prime}}^{+}\left(t^{\prime}\right)\right\rangle,
\end{aligned}
$$

where $T$ and $\tilde{T}$ denote time-ordering and anti-time ordering of the Heisenberg operators $d_{\sigma}(t), \ldots, b_{\sigma}^{+}(t)$. We use the notifications of [2]. For the brevity we use $\lambda$ instead $\lambda, \sigma$ in Eqs. (6). Only two of four Green's functions (5) and (6) are independent because of the linearly dependence:

$$
G^{-+}+G^{+-}=G^{--}+G^{++} \text {. }
$$

The analogous equations exist for the $\mathcal{G}$ Green's functions. The retarded and advanced Green's functions are given by

$$
\begin{aligned}
& G^{r}=G^{--}-G^{-+}, \quad G^{a}=G^{--}-G^{+-}, \\
& G^{r}\left(t, t^{\prime}\right)=-i \theta\left(t-t^{\prime}\right)\left\langle\left\{d(t), d^{+}\left(t^{\prime}\right)\right\}\right\rangle, \\
& G^{a}\left(t, t^{\prime}\right)=i \theta\left(t^{\prime}-t\right)\left\langle\left\{d(t), d^{+}\left(t^{\prime}\right)\right\}\right\rangle,
\end{aligned}
$$

where the curly brackets denote anticommutators.

\section{Correlation function for Anderson impurity model}

Because the unperturbed Hamiltonian (2) includes Coulomb interaction the ordinary Wick theorem is inapplicable and generalized Wick theorem (GWT) must be used [4-7]. GWT contains new diagrammatical elements named irreducible Green's functions, which have the structure of $\mathrm{Ku}-$ bo comulants and contain all charge, spin and pairing fluctuations. For example in simplest case we have

$$
\begin{gathered}
\left\langle T d_{\sigma_{1}}\left(t_{1}\right) d_{\sigma_{2}}\left(t_{2}\right) d_{\sigma_{3}}^{+}\left(t_{3}\right) d_{\sigma_{4}}^{+}\left(t_{4}\right)\right\rangle_{0}= \\
=\left\langle T d_{\sigma_{1}}\left(t_{1}\right) d_{\sigma_{4}}^{+}\left(t_{4}\right)\right\rangle_{0}\left\langle T d_{\sigma_{2}}\left(t_{2}\right) d_{\sigma_{3}}^{+}\left(t_{3}\right)\right\rangle_{0}- \\
-\left\langle T d_{\sigma_{1}}\left(t_{1}\right) d_{\sigma_{3}}^{+}\left(t_{3}\right)\right\rangle_{0}\left\langle T d_{\sigma_{2}}\left(t_{2}\right) d_{\sigma_{4}}^{+}\left(t_{4}\right)\right\rangle_{0}+ \\
+G_{2}^{(0) i r r}\left[\sigma_{1} t_{1}, \sigma_{2} t_{2} \mid \sigma_{3} t_{3}, \sigma_{4} t_{4}\right],
\end{gathered}
$$

where $G_{1}^{(0) i r r}$ is the two-particle irreducible Greens function which must be found. This question is the special problem of strongly correlated electron system and has been studied in many papers [8-17].

The pieces of the Hamiltonian describe the tunneling coupling between the contacts and interacting central region. The current from left contact through the left barrier to the central is [18-22]

$$
\begin{aligned}
& I_{L}^{(t)}=-e\left\langle\dot{N}_{L}\right\rangle=-\frac{i e}{\hbar}\left\langle\left[H, N_{L}\right]\right\rangle, \\
& N_{L}=\sum_{\mathbf{k}, \lambda=\mathbf{L}, \sigma} C_{\mathbf{k} \lambda \sigma}^{+} C_{\mathbf{k} \lambda \sigma},
\end{aligned}
$$

with result

$$
\begin{gathered}
I_{L}=\frac{i e}{\hbar} \sum_{\mathbf{k}, \lambda=\mathbf{L}, \sigma}\left(V_{\mathbf{k} \lambda \sigma}\left\langle C_{\mathbf{k} \lambda \sigma}^{+} d_{\sigma}\right\rangle-V_{\mathbf{k} \lambda \sigma}^{*}\left\langle d_{\sigma}^{+} C_{\mathbf{k} \lambda \sigma}\right\rangle\right)= \\
=\frac{i e}{\hbar} \sum_{\lambda \sigma}\left(\left\langle b_{\lambda \sigma}^{+} d_{\sigma}\right\rangle-\left\langle d_{\sigma}^{+} b_{\lambda \sigma}\right\rangle\right) .
\end{gathered}
$$

Two Green's functions are present

$$
\begin{aligned}
& G_{\sigma, \mathbf{k} \lambda}^{-+}\left(t, t^{\prime}\right)=i\left\langle C_{\mathbf{k} \lambda \sigma}^{+}\left(t^{\prime}\right) d_{\sigma}(t)\right\rangle, \\
& G_{\mathbf{k} \lambda, \sigma}^{-+}\left(t, t^{\prime}\right)=i\left\langle d_{\sigma}^{+}\left(t^{\prime}\right) C_{\mathbf{k} \lambda \sigma}(t)\right\rangle,
\end{aligned}
$$

which must be calculated by using Keldysh formalism.

As a result of the existence of the irreducible Green's functions is the appearance of a new element of diagrammatic theory of strongly correlated electron systems, the so-called correlation function, which is the infinite sum of strong connected irreducible Green's functions. This quantity marked by us with letter $\Lambda$ determines the Dyson-type equation of strongly correlated systems.

In Matsubara theory of equilibrium systems this Dysontype equation has the form

$$
G=\Lambda+\Lambda \mathcal{G}_{0} G
$$

where $G$ is the full renormalized Green's function of strong interacted localized electrons, $\Lambda$ is the correlation function and $\mathcal{G}_{0}$ is the bare propagator of free itinerant electrons.

Correlation function differs from mass operator of the weak coupling field theory because this last quantity begins and ends with zero order propagators, but $\Lambda$ could have not them.

In Keldysh formalism we use matrix Green's functions composed from different elements of time evolution in contour space. We have the matrices

$$
\hat{G}=\left(\begin{array}{ll}
G^{--} & G^{-+} \\
G^{+-} & G^{++}
\end{array}\right), \quad \hat{\Lambda}=\left(\begin{array}{cc}
\Lambda^{--} & \Lambda^{-+} \\
\Lambda^{+-} & \Lambda^{++}
\end{array}\right),
$$

and also such one for the free propagator $\mathcal{G}_{0}$.

Dyson-type equation for nonequilibrium strong correlated electron systems has the form

$$
\hat{G}=\hat{\Lambda}+\hat{\Lambda} \hat{\mathcal{G}}_{0} \hat{G}
$$

Besides the Eq. (7) we have the relations

$$
\begin{aligned}
& \Lambda^{--}+\Lambda^{++}=\Lambda^{-+}+\Lambda^{+-} \\
& \mathcal{G}_{0}^{--}+\mathcal{G}_{0}^{++}=-\left(\mathcal{G}_{0}^{-+}+\mathcal{G}_{0}^{+-}\right),
\end{aligned}
$$


and the definitions of retarded and advanced functions

$$
\begin{aligned}
& G^{r}=G^{--}-G^{-+}=G^{+-}-G^{++}, \\
& G^{a}=G^{--}-G^{+-}=G^{-+}-G^{++}, \\
& \mathcal{G}_{0}^{r}=\mathcal{G}_{0}^{--}+\mathcal{G}_{0}^{-+}=-\left(\mathcal{G}_{0}^{+-}+\mathcal{G}_{0}^{++}\right), \\
& \mathcal{G}_{0}^{a}=\mathcal{G}_{0}^{--}+\mathcal{G}_{0}^{+-}=-\left(\mathcal{G}_{0}^{-+}+\mathcal{G}_{0}^{++}\right), \\
& \Lambda^{r}=\Lambda^{--}-\Lambda^{-+}=\Lambda^{+-}-\Lambda^{++}, \\
& \Lambda^{a}=\Lambda^{--}-\Lambda^{+-}=\Lambda^{-+}-\Lambda^{++} .
\end{aligned}
$$

The next section is devoted to nonequilibrium perturbative theory of strongly correlated electron systems.

\section{Nonequilibrium perturbative theory for Anderson model}

The nonequilibrium perturbative theory obtains the thermal averages by assuming that we can know only the state at $t=-\infty$, because initially, at $t=-\infty$, the system is equilibrium. The perturbation is turned on at $t=-\infty$ and introduced adiabatical as stationary nonequilibrium at $t=0$. After that the perturbation is taken away adiabatically and vanishes at $t=\infty$. The state at $t=\infty$ cannot be well defined $[23,24]$. The time evolution is realized along real-time contour which starts and ends at $t=-\infty$ as illustrated in Fig. 1.

The thermal average in the Heisenberg representation at $t=0$ can be obtained in the form [2]

$$
\left\langle A\left(t, t^{\prime}\right)\right\rangle=\left\langle\hat{S}^{-1} T \widetilde{A}\left(t, t^{\prime}\right) \hat{S}\right\rangle
$$

where

$$
\begin{gathered}
\hat{S}=\hat{S}(\infty,-\infty)=T \exp \left(-i \int_{-\infty}^{\infty} \widetilde{H}_{i}(t) d t\right) \\
\hat{S}^{-1}=\hat{S}^{+}=\widetilde{T} \exp \left(i \int_{-\infty}^{\infty} \widetilde{H}_{i}(t) d t\right) .
\end{gathered}
$$

The expansions of these exponents give the forms [3]

$$
\begin{aligned}
\left\langle A\left(t, t^{\prime}\right)\right\rangle= & \sum_{n=0}^{\infty} \sum_{m=0}^{\infty} \frac{1}{n !} \frac{1}{m !}\left(\frac{i}{\hbar}\right)^{n}\left(\frac{-i}{\hbar}\right)^{m} \int_{-\infty}^{\infty} d t_{1} \ldots \int_{-\infty}^{\infty} d t_{n} \int_{-\infty}^{\infty} d t_{1}^{\prime} \ldots \\
& \ldots \int_{-\infty}^{\infty} d t_{m}^{\prime}\left\langle\left\{\widetilde{T} \widetilde{H}_{i}\left(t_{1}^{+}\right) \ldots \widetilde{H}_{i}\left(t_{n}^{+}\right)\right\} \times\right. \\
\times & \left.\left\{T \widetilde{H}_{i}\left(t_{m}^{\prime-}\right) \ldots \widetilde{H}_{i}\left(t_{m}^{\prime-}\right) \widetilde{A}\left(t^{-}, t^{\prime+}\right)\right\}\right\rangle_{\mathrm{av}}
\end{aligned}
$$

where $\langle\cdots\rangle_{\mathrm{av}}=\operatorname{Tr}(\widetilde{\rho}(-\infty) \ldots)$ and operators with tilde $\widetilde{H}_{i}$ are in the interaction representation. Then the thermal averages are derived by using GWT.

We use Hamiltonian without time dependence because the states are supposed stationary.

In the next part of the paper we shall use two forms of operator $A$ :

$$
\begin{aligned}
& A_{1}\left(t, t^{\prime}\right)=i b_{\lambda \sigma}^{+}\left(t^{\prime+}\right) d_{\sigma}\left(t^{-}\right), \\
& A_{2}\left(t, t^{\prime}\right)=i d_{\sigma}^{+}\left(t^{\prime+}\right) b_{\lambda \sigma}\left(t^{-}\right),
\end{aligned}
$$

and determine the Green's functions

$$
\begin{aligned}
& G_{\sigma, \lambda \sigma^{\prime}}^{-+}\left(t, t^{\prime}\right)=i\left\langle b_{\lambda \sigma^{\prime}}^{+}\left(t^{\prime+}\right) d_{\sigma}\left(t^{-}\right)\right\rangle, \\
& G_{\lambda \sigma, \sigma^{\prime}}^{-+}\left(t, t^{\prime}\right)=i\left\langle d_{\sigma}^{+}\left(t^{\prime+}\right) b_{\lambda \sigma}\left(t^{-}\right)\right\rangle .
\end{aligned}
$$

We shall take the odd terms of perturbation Eqs. (20) and maintain the first two nonzero contributions. First of them is

$$
A^{(1)}\left(t, t^{\prime}\right)=
$$

$=\int_{-\infty}^{\infty} d t_{1}\left\langle i \widetilde{T} \widetilde{H}_{i}\left(t_{1}^{+}\right) \widetilde{A}\left(t^{-}, t^{++}\right)-i T \widetilde{A}\left(t^{-}, t^{++}\right) \widetilde{H}_{i}\left(t_{1}^{-}\right)\right\rangle$,

and the third contribution is

$$
\begin{gathered}
A^{(3)}\left(t, t^{\prime}\right)=\iint_{-\infty}^{\infty} \int d t_{1} d t_{2} d t_{3}\left\{\frac{(-i)^{3}}{3 !}\left\langle T \widetilde{A}\left(t^{-}, t^{\prime}\right) \widetilde{H}_{i}\left(t_{1}^{-}\right) \widetilde{H}_{i}\left(t_{2}^{-}\right) \widetilde{H}_{i}\left(t_{3}^{-}\right)\right\rangle+\right. \\
+\frac{(i)}{1 !} \frac{(-i)^{2}}{2 !}\left\langle\widetilde{T} \widetilde{H}_{i}\left(t_{1}^{+}\right) T \widetilde{A}\left(t^{-}, t^{\prime}\right) \widetilde{H}_{i}\left(t_{2}^{-}\right) \widetilde{H}_{i}\left(t_{3}^{-}\right)\right\rangle+\frac{(i)^{2}}{2 !} \frac{(-i)}{1 !}\left\langle\widetilde{T}_{i}\left(t_{1}^{+}\right) \widetilde{H}_{i}\left(t_{2}^{+}\right) \widetilde{A}\left(t^{-}, t^{\prime+}\right) \widetilde{H}_{i}\left(t_{3}^{-}\right)\right\rangle+ \\
\left.+\frac{(i)^{3}}{3 !}\left\langle\widetilde{T} \widetilde{H}_{i}\left(t_{1}^{+}\right) \widetilde{H}_{i}\left(t_{2}^{+}\right) \widetilde{H}_{i}\left(t_{3}^{+}\right) T \widetilde{A}\left(t^{-}, t^{\prime+}\right)\right\rangle\right\} .
\end{gathered}
$$


We have devided the pertubation operator $H_{i}$ in two parts

$$
H_{i}=H_{i 1}+H_{i 2}, \quad H_{i 1}=\sum_{\lambda \sigma} b_{\lambda \sigma}^{+} d_{\sigma}, \quad H_{i 2}=\sum_{\lambda \sigma} d_{\sigma}^{+} b_{\lambda \sigma}
$$

and obtain the more simple forms

$$
\begin{aligned}
& A_{1}^{(1)}\left(t, t^{\prime}\right)=\int_{-\infty}^{\infty}\left\langle i \widetilde{T} \widetilde{H}_{i 2}\left(t_{1}^{+}\right) \widetilde{A}_{1}\left(t^{-}, t^{\prime+}\right)-i T \widetilde{A}_{1}\left(t^{-}, t^{\prime+}\right) \widetilde{H}_{i 2}\left(t_{1}^{-}\right)\right\rangle d t_{1}, \\
& A_{2}^{(1)}\left(t, t^{\prime}\right)=\int_{-\infty}^{\infty}\left\langle i \widetilde{T} \widetilde{H}_{i 1}\left(t_{1}^{+}\right) \widetilde{A}_{2}\left(t^{-}, t^{\prime+}\right)-i T \widetilde{A}_{2}\left(t^{-}, t^{+}\right) \widetilde{H}_{i 1}\left(t_{1}^{-}\right)\right\rangle d t_{1},
\end{aligned}
$$

and

$$
\begin{aligned}
A_{1}^{(3)}\left(t, t^{\prime}\right)= & \iint_{-\infty}^{\infty} \int d t_{1} d t_{2} d t_{3} i\left\{\frac{1}{2}\left\langle T \widetilde{A}_{1}\left(t^{-}, t^{\prime+}\right) \widetilde{H}_{i 1}\left(t_{1}^{-}\right) \widetilde{H}_{i 2}\left(t_{2}^{-}\right) \widetilde{H}_{i 2}\left(t_{3}^{-}\right)\right\rangle-\frac{1}{2}\left\langle\widetilde{T} \widetilde{H}_{i 1}\left(t_{1}^{+}\right) T \widetilde{A}_{1}\left(t^{-}, t^{t^{+}}\right) \widetilde{H}_{i 2}\left(t_{2}^{-}\right) \widetilde{H}_{i 2}\left(t_{3}^{-}\right)\right\rangle-\right. \\
& -\left\langle\widetilde{T} \widetilde{H}_{i 2}\left(t_{1}^{+}\right) T \widetilde{A}_{1}\left(t^{-}, t^{++}\right) \widetilde{H}_{i 1}\left(t_{2}^{-}\right) \widetilde{H}_{i 2}\left(t_{3}^{-}\right)\right\rangle+\left\langle\widetilde{T} \widetilde{H}_{i 1}\left(t_{1}^{+}\right) H_{i 2}\left(t_{2}^{+}\right) \widetilde{A}_{1}\left(t^{-}, t^{++}\right) \widetilde{H}_{i 2}\left(t_{3}^{-}\right)\right\rangle+ \\
+ & \left.\frac{1}{2}\left\langle\widetilde{T} \widetilde{H}_{i 2}\left(t_{1}^{+}\right) \widetilde{H}_{i 2}\left(t_{2}^{+}\right) T \widetilde{A}_{1}\left(t^{-}, t^{\prime+}\right) \widetilde{H}_{i 1}\left(t_{3}^{-}\right)\right\rangle-\frac{1}{2}\left\langle\widetilde{T}_{i 1}\left(t_{1}^{+}\right) H_{i 2}\left(t_{2}^{+}\right) \widetilde{H}_{i 2}\left(t_{3}^{+}\right) T \widetilde{A}_{1}\left(t^{-}, t^{\prime+}\right)\right\rangle\right\} .
\end{aligned}
$$

Analogous equations can be obtained for $A_{2}^{(3)}\left(t, t^{\prime}\right)$ by changing indices 1 and 2 in (27).

The result of calculation $A_{1}^{(1)}$ and $A_{2}^{(1)}$ can be presented as a sum of two contributions depicted on the Fig. 2:

$$
\begin{aligned}
A_{1}^{(1)}\left(t, t^{\prime}\right)= & \int_{-\infty}^{\infty} d t_{1} \sum_{\lambda_{1} \sigma_{1}}\left[G_{\sigma \sigma_{1}}^{--}\left(t, t_{1}\right) \mathcal{G}_{\lambda_{1} \sigma_{1}, \lambda \sigma}^{-+}\left(t_{1}, t^{\prime}\right)-\right. \\
& \left.-G_{\sigma \sigma_{1}}^{-+}\left(t, t_{1}\right) \mathcal{G}_{\lambda_{1} \sigma_{1}, \lambda \sigma}^{++}\left(t_{1}, t^{\prime}\right)\right], \\
A_{2}^{(1)}\left(t, t^{\prime}\right)= & \int_{-\infty}^{\infty} d t_{1} \sum_{\lambda_{1} \sigma_{1}}\left[\mathcal{G}_{\lambda \sigma, \lambda_{1} \sigma_{1}}^{--}\left(t, t_{1}\right) G_{\sigma_{1} \sigma}^{-+}\left(t_{1}, t^{\prime}\right)-\right. \\
& \left.-\mathcal{G}_{\lambda \sigma, \lambda_{1} \sigma_{1}}^{-+}\left(t, t_{1}\right) G_{\sigma_{1} \sigma}^{++}\left(t_{1}, t^{\prime}\right)\right] .
\end{aligned}
$$

(a)

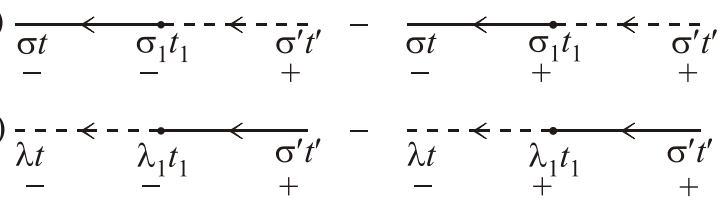

Fig. 2. Diagrams of first order of perturbation theory: for $A_{1}^{(1)}$ (a) and for $A_{2}^{(1)}$ (b). Thin solid line is $G$ propagator and dashed line is $\mathcal{G}$ propagator.
In matrix representation determined by Eqs. (14) we have

$$
\begin{aligned}
& A_{1}^{(1)}\left(t, t^{\prime}\right)=\int_{-\infty}^{\infty} d t_{1} \sum_{\lambda_{1} \sigma_{1}}\left(\hat{G}_{\sigma_{\sigma_{1}}}\left(t, t_{1}\right) \hat{\sigma}_{3} \hat{\mathcal{G}}_{\lambda_{1} \sigma_{1}, \lambda \sigma}\left(t_{1}, t^{\prime}\right)\right)^{-+}, \\
& A_{2}^{(1)}\left(t, t^{\prime}\right)=\int_{-\infty}^{\infty} d t_{1} \sum_{\lambda_{1} \sigma_{1}}\left(\hat{\mathcal{G}}_{\lambda \sigma, \lambda_{1} \sigma_{1}}\left(t, t_{1}\right) \hat{\sigma}_{3} \hat{G}_{\sigma_{1} \sigma}\left(t_{1}, t^{\prime}\right)\right)^{-+},
\end{aligned}
$$

where $\sigma_{3}$ is the Pauli matrix. The third order of perturbation theory contains the iteration of these last formulas and some more contributions obliged to the existence of the correlation function. The iterative contributions of this order are

$$
\begin{aligned}
& A_{1}^{(3) \text { it }}\left(t, t^{\prime}\right)=\iint_{-\infty}^{\infty} \int d t_{1} d t_{2} d t_{3} \sum_{\lambda_{1} \sigma_{1} \ldots \lambda_{3} \sigma_{3}}\left(\hat{G}_{\sigma_{\sigma_{1}}}\left(t, t_{1}\right) \hat{\sigma}_{3} \times\right. \\
& \left.\times \hat{\mathcal{G}}_{\lambda_{1} \sigma_{1}, \lambda_{2} \sigma_{2}}\left(t_{1}, t_{2}\right) \hat{\sigma}_{3} \hat{G}_{\sigma_{2} \sigma_{3}}\left(t_{2}, t_{3}\right) \hat{\sigma}_{3} \hat{\mathcal{G}}_{\lambda_{3} \sigma_{3}, \lambda^{\prime} \sigma^{\prime}}\left(t_{3}, t^{\prime}\right)\right)^{-+}, \\
& A_{2}^{(3) \text { it }}\left(t, t^{\prime}\right)=\iint_{-\infty}^{\infty} \int d t_{1} d t_{2} d t_{3} \sum_{\lambda_{1} \sigma_{1} \ldots \lambda_{3} \sigma_{3}}\left(\hat{\mathcal{G}}_{\lambda \sigma, \lambda_{1} \sigma_{1}}\left(t, t_{1}\right) \hat{\sigma}_{3} \times\right. \\
& \left.\times \hat{G}_{\sigma_{1} \sigma_{2}}\left(t_{1}, t_{2}\right) \hat{\sigma}_{3} \hat{\mathcal{G}}_{\lambda_{2} \sigma_{2}, \lambda_{3} \sigma_{3}}\left(t_{2}, t_{3}\right) \hat{\sigma}_{3} \hat{G}_{\sigma_{3} \sigma^{\prime}}\left(t_{3}, t^{\prime}\right)\right)^{-+},
\end{aligned}
$$

and the irreducible contribution for the $A_{1}^{(3)}$ has the form 


$$
\begin{aligned}
& A_{1}^{(3) i r r}\left(t, t^{\prime}\right)=\iint_{-\infty}^{\infty} \int_{0} d t_{1} d t_{2} d t_{3} \sum_{\lambda_{1} \sigma_{1} \ldots \lambda_{3} \sigma_{3}}\left\{\left\langle T d_{\sigma}\left(t^{-}\right) d_{\sigma_{1}}\left(t_{1}^{-}\right) d_{\sigma_{2}}^{+}\left(t_{2}^{-}\right) d_{\sigma_{3}}^{+}\left(t_{3}^{-}\right)\right\rangle^{\mathrm{irr}}\left[\mathcal{G}_{\lambda_{2} \sigma_{2}, \lambda_{1} \sigma_{1}}^{--}\left(t_{2}^{-}, t_{1}^{-}\right) \mathcal{G}_{\lambda_{3} \sigma_{3}, \lambda^{\prime} \sigma^{\prime}}^{-+}\left(t_{3}^{-}, t^{\prime^{+}}\right)\right]-\right. \\
& -\left\langle\widetilde{T} d_{\sigma}\left(t^{-}\right) d_{\sigma_{1}}\left(t_{1}^{+}\right) d_{\sigma_{2}}^{+}\left(t_{2}^{-}\right) d_{\sigma_{3}}^{+}\left(t_{3}^{-}\right)\right\rangle^{\text {irr }}\left[\mathcal{G}_{\lambda_{2} \sigma_{2}, \lambda_{1} \sigma_{1}}^{-+}\left(t_{2}^{-}, t_{1}^{+}\right) \mathcal{G}_{\lambda_{3} \sigma_{3}, \lambda^{\prime} \sigma^{\prime}}^{-+}\left(t_{3}^{-}, t^{\prime+}\right)\right]+ \\
& +\left\langle\widetilde{T} d_{\sigma_{2}}^{+}\left(t_{2}^{+}\right) T d_{\sigma}\left(t^{-}\right) d_{\sigma_{1}}\left(t_{1}^{-}\right) d_{\sigma_{3}}^{+}\left(t_{3}^{-}\right)\right\rangle^{\mathrm{irr}}\left[-\mathcal{G}_{\lambda_{2} \sigma_{2}, \lambda_{1} \sigma_{1}}^{+-}\left(t_{2}^{+}, t_{1}^{-}\right) \mathcal{G}_{\lambda_{3} \sigma_{3}, \lambda^{\prime} \sigma^{\prime}}^{-+}\left(t_{3}^{-}, t^{\prime+}\right)\right]+ \\
& +\left\langle\widetilde{T} d_{\sigma_{3}}^{+}\left(t_{3}^{+}\right) T d_{\sigma}\left(t^{-}\right) d_{\sigma_{1}}\left(t_{1}^{-}\right) d_{\sigma_{2}}^{+}\left(t_{2}^{-}\right)\right\rangle^{\mathrm{irr}}\left[\mathcal{G}_{\lambda_{3} \sigma_{3}, \lambda^{\prime} \sigma^{\prime}}^{++}\left(t_{3}^{+}, t^{\prime+}\right) \mathcal{G}_{\lambda_{2} \sigma_{2}, \lambda_{1} \sigma_{1}}^{--}\left(t_{2}^{-}, t_{1}^{-}\right)\right]- \\
& -\left\langle T d_{\sigma_{3}}^{+}\left(t_{3}^{+}\right) d_{\sigma_{2}}^{+}\left(t_{2}^{+}\right) T d_{\sigma}\left(t^{-}\right) d_{\sigma_{1}}\left(t_{1}^{-}\right)\right\rangle^{\mathrm{irr}}\left[\mathcal{G}_{\lambda_{3} \sigma_{3}, \lambda^{\prime} \sigma^{\prime}}^{++}\left(t_{3}^{+}, t^{\prime+}\right) \mathcal{G}_{\lambda_{2} \sigma_{2}, \lambda_{1} \sigma_{1}}^{+-}\left(t_{2}^{+}, t_{1}^{-}\right)\right]+ \\
& +\left\langle\widetilde{T} d_{\sigma_{1}}\left(t_{1}^{+}\right) d_{\sigma_{2}}^{+}\left(t_{2}^{+}\right) T d_{\sigma}\left(t^{-}\right) d_{\sigma_{3}}^{+}\left(t_{3}^{-}\right)\right\rangle^{\mathrm{irr}}\left[\mathcal{G}_{\lambda_{2} \sigma_{2}, \lambda_{1} \sigma_{1}}^{++}\left(t_{2}^{+}, t_{1}^{+}\right) \mathcal{G}_{\lambda_{3} \sigma_{3}, \lambda^{\prime} \sigma^{\prime}}^{-+}\left(t_{3}^{-}, t^{++}\right)\right]- \\
& -\left\langle\widetilde{T} d_{\sigma_{1}}\left(t_{1}^{+}\right) d_{\sigma_{3}}^{+}\left(t_{3}^{+}\right) T d_{\sigma}\left(t^{-}\right) d_{\sigma_{2}}^{+}\left(t_{2}^{-}\right)\right\rangle^{\mathrm{irr}}\left[\mathcal{G}_{\lambda_{2} \sigma_{2}, \lambda_{1} \sigma_{1}}^{-+}\left(t_{2}^{-}, t_{1}^{+}\right) \mathcal{G}_{\lambda_{3} \sigma_{3}, \lambda^{\prime} \sigma^{\prime}}^{++}\left(t_{3}^{+}, t^{\prime+}\right)\right]+ \\
& \left.+\left\langle\widetilde{T} d_{\sigma_{1}}\left(t_{1}^{+}\right) d_{\sigma_{2}}^{+}\left(t_{2}^{+}\right) d_{\sigma_{3}}^{+}\left(t_{3}^{+}\right) d_{\sigma}\left(t^{-}\right)\right\rangle^{\mathrm{irr}}\left[\mathcal{G}_{\lambda_{2} \sigma_{2}, \lambda_{1} \sigma_{1}}^{++}\left(t_{2}^{+}, t_{1}^{+}\right) \mathcal{G}_{\lambda_{3} \sigma_{3}, \lambda^{\prime} \sigma^{\prime}}^{++}\left(t_{3}^{+}, t^{\prime+}\right)\right]\right\} .
\end{aligned}
$$

The contributions which belong $A_{1}^{(3) i r r}$ and $A_{2}^{(3) i r r}$ are depicted on the upper and lower parts of Fig. 3, correspondingly.
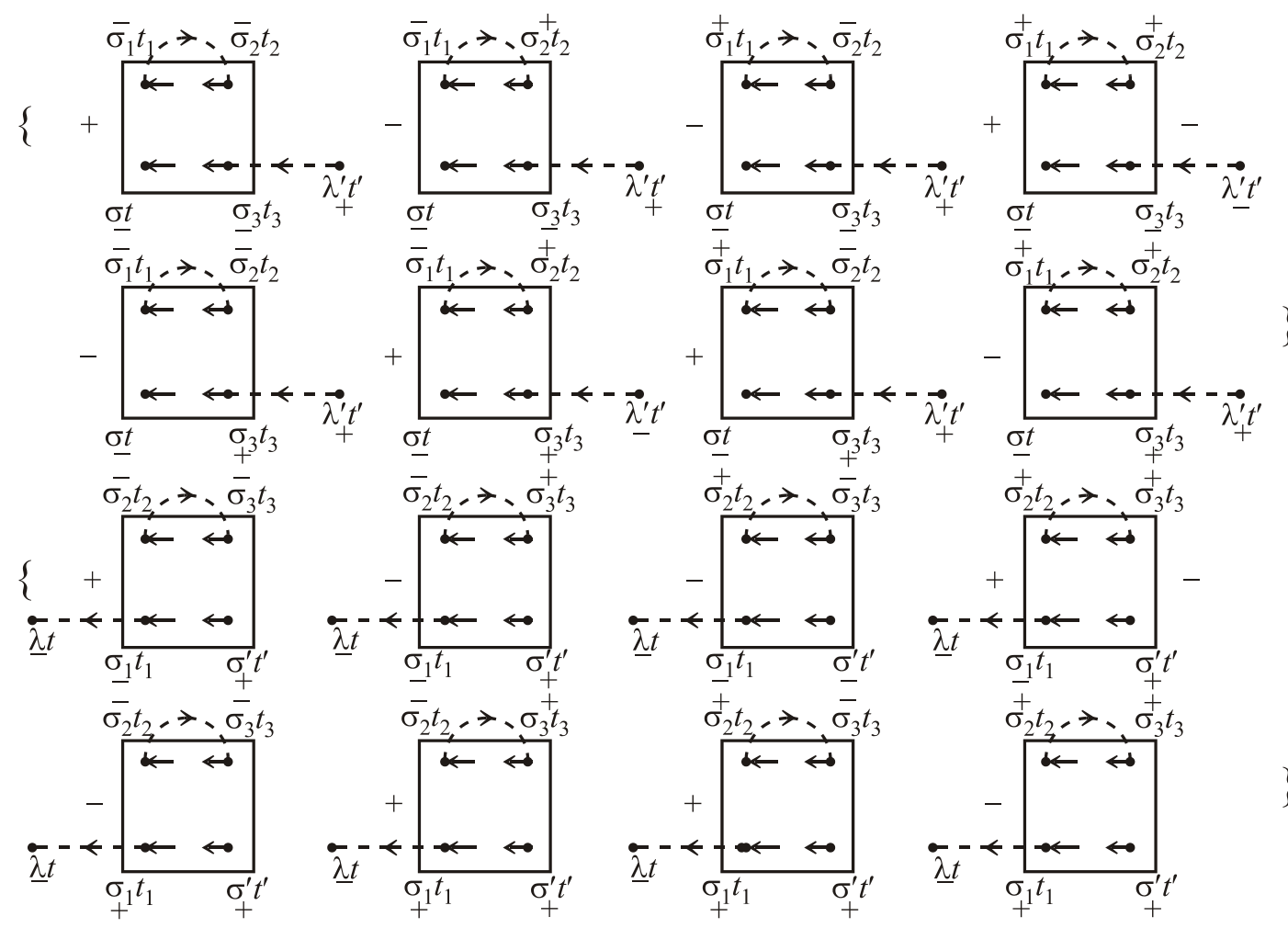

Fig. 3. In the upper part are depicted irreducible diagrams which belong to the $A_{1}^{(3) i r r}$ and in the lower part are the diagrams which belong to $A_{2}^{(3) \text { irr }}$. The rectangles depict irreducible Green's functions and thin dashed lines depict $\mathcal{G}$ Green's functions. 


\section{Conclusions}

The nonequilibrium theory of the strongly correlated electron systems is proposed. We take into account nonapplicability of the ordinary Wick theorem for strongly correlated systems and use the new generalized Wick theorem, which is used to obtain the termal averages of the product of fermion operators. GWT takes into account the additional contribution of the Kubo cumulants.

We use the Keldysh formalism of contour time evolution with additional number of the one particle Green's functions and obtain the current from left contact through the left barrier to the central interactions region.

New elements of diagrammatic theory of our strongly correlated systems are the irreducible Green's functions or Kubo cumulants.

We develop the perturbation theory about the small hybridization matrix element and obtain the correction for the current value.

1. L.V. Keldysh, Sov. Phys. JETP 20, 1018 (1965).

2. E.M. Lifshitz and L.P. Pitaevskii, Physical Kinetics, Pergamon Press, Oxford (1981).

3. Akira Oguri, J. Phys. Soc. Jpn. 71, 2969 (2008).

4. M.I. Vladimir and V.A. Moskalenko, Teor. Mat. Fiz. 82, 428 (1990) [Theor. Math. Phys. 82, 301 (1990)].

5. S.I. Vakaru, M.I. Vladimir, and V.A. Moskalenko, Teor. Mat. Fiz. 85, 248 (1990) [Theor. Math. Phys. 85, 1185 (1990)].

6. N.N. Bogoliubov and V.A. Moskalenko, Teor. Mat. Fiz. 86, 16 (1991) [Theor. Math. Phys. 86, 10 (1991)].

7. N.N. Bogoliubov and V.A. Moskalenko, Teor. Mat. Fiz. 92, 182 (1992) [Theor. Math. Phys. 92, 820 (1992)].

8. V.A. Moskalenko, P. Entel, and D.F. Digor, Phys. Rev. B 59, 619 (1999); Physica B: Condens. Matter 259-261, 781 (1999).
9. V.A. Moskalenko, P. Entel, M. Marinaro, N.B. Perkins, and C. Holfort, Phys. Rev. B 63, 245119 (2001).

10. V.A. Moskalenko, P. Entel, M. Marinaro, D.F. Digor, and M.I. Vladimir, Mold. J. Phys. Sci. 2, No. 2, 132 (2003).

11. V.A. Moskalenko, D.F. Digor, P. Entel, M. Marinaro and N.B. Perkins, Models for Strongly Interacting Fermion Systems. Models and Methods of High- $T_{C}$ Superconductivity: some Frontal Aspects, New York (2003), Vol. 2 [ch. 6], p. 201 (Horizons in World Physics, 242).

12. V.A. Moskalenko, P. Entel, M. Marinaro, and D.F. Digor, JETP 97, 632 (2003).

13. V.A. Moskalenko, P. Entel, D.F. Digor, and L.A. Dohotaru, Phase Transitions 78, 277 (2005).

14. V.A. Moskalenko, P. Entel and D.F. Digor, Physics of Particles and Nuclei 36, Suppl. 1, 100 (2005).

15. V.A. Moskalenko, P. Entel, and D.F. Digor, Fiz. Nizk. Temp. 32, 609 (2006) [Low Temp. Phys. 32, 462 (2006)].

16. V.A. Moskalenko, P. Entel, and D.F. Digor, Phys. Rev. B 74, 075109 (2006).

17. V.A. Moskalenko, P. Entel, D.F. Digor, L.A. Dohotaru, and R. Citro, Teor. Mat. Fiz. 155, 474 (2008) [Theor. Math. Phys. 155, 914 (2008)].

18. Yigal Meir and Ned S. Wingreen, Phys. Rev. Lett. 68, 2812 (1992).

19. Ned S. Wingreen, Karsten W. Iacobson, and John W. Wilkins, Phys. Rev. B 40, 11834 (1989).

20. Selman Hershfield, John H. Davces, and John W. Wilkins, Phys. Rev. B 46, 7046 (1992).

21. Ned S. Wingreen, Antti-Pekka Jauho, and Vigal Meir, Phys. Rev. B 48, 8487 (1993).

22. Antti-Pekka Jauho and John Yigal Meir, Phys. Rev. B 50, 5528 (1994).

23. J. Schwinger, J. Math. Phys. (N.Y.) 2, 407 (1961).

24. Mami Hamasaki, arXiv: cond-mat/0506752v5 (2006). 